\begin{tabular}{|c|c|c|}
\hline Case Reports in & \multicolumn{2}{|c|}{ Case Rep Gastroenterol 2015;9:206-214 } \\
\hline Gastroenterology & $\begin{array}{l}\text { DOI: } 10.1159 / 000431308 \\
\text { Publisnea onime: June } 11,2015\end{array}$ & $\begin{array}{l}\text { (c) } 2015 \text { S. Karger AG, Basel } \\
1662-0631 / 15 / 0092-0206 \$ 39.50 / 0 \\
\text { www.karger.com/crg }\end{array}$ \\
\hline & \multicolumn{2}{|c|}{$\begin{array}{l}\text { This is an Open Access article licensed under the terms of the Creative Commons } \\
\text { Attribution-NonCommercial } 3.0 \text { Unported license (CC BY-NC) (www.karger.com/OA- } \\
\text { license), applicable to the online version of the article only. Distribution permitted for non- } \\
\text { commercial purposes only. }\end{array}$} \\
\hline
\end{tabular}

\title{
Impact of Endoscopic Ultrasound Procedures in Various Pancreatobiliary Disorders in Indonesia Based on a Case Series in a Private Hospital
}

\author{
C. Rinaldi A. Lesmana ${ }^{a, b} \quad$ Khek Yu Hoc Laurentius A. Lesmana ${ }^{\mathrm{b}}$ \\ ${ }^{a}$ Hepatobiliary Division, Department of Internal Medicine, Cipto Mangunkusumo Hospital, \\ and ${ }^{b}$ Digestive Disease and GI Oncology Center, Medistra Hospital, Jakarta, Indonesia; \\ 'Division of Gastroenterology and Hepatology, Department of Medicine, National \\ University Hospital, Singapore
}

\section{Key Words}

Endoscopic ultrasound - Endoscopic retrograde cholangiopancreatography · Pancreatobiliary disorders · Developing countries

\begin{abstract}
Background: Endoscopic retrograde cholangiopancreatography (ERCP) is a common technique for assessing the pancreas and the biliary system; however, the potential complications have raised concern among endoscopists and patients. Recently, the need of endoscopic ultrasound (EUS) as an additional tool of assessment before the ERCP procedure has been increasing. The need of EUS in developing countries is still a matter of debate regarding the cost, investment, and training. Here, we report the significant impact of EUS on several unselected interesting cases of pancreatobiliary disorders. Method: We selected several interesting cases from the patients who underwent EUS at our private hospital in Jakarta, Indonesia. The EUS procedures were performed by one experienced endosonographer and one EUS trainee who are very experienced with transabdominal ultrasound. The equipment was an Olympus JF UCT 180 EUS scope which was connected to an Aloka IPF-1701C ultrasound machine (Tokyo, Japan). Results: Five interesting cases were included from patients who underwent EUS due to pancreatobiliary disorders. The cases included recurrent pancreatitis due to pancreatic stone at the small branch that obstructed the main pancreatic duct, common bile
\end{abstract}


Lesmana et al.: Impact of Endoscopic Ultrasound Procedures in Various

Pancreatobiliary Disorders in Indonesia Based on a Case Series in a Private Hospital

duct (CBD) stone with insignificant duct dilatation, pancreatic head cancer with total obstruction at the distal CBD and portal vein infiltration, pancreas divisum in a young girl, and distal CBD mass that caused obstructive jaundice. Conclusions: The EUS procedure has shown a significant impact in managing patients with pancreatobiliary diseases. In most developing countries, EUS needs to be evaluated further regarding the cost, investment, and training.

(c) 2015 S. Karger AG, Basel

\section{Background}

Endoscopic retrograde cholangiopancreatography (ERCP) was introduced in 1968 as a safe and accurate technique for assessing the pancreas and the biliary system. In the past, ERCP was used both in diagnostic and therapeutic procedures [1, 2]. However, the potential complications or side effects from the procedure itself and also the failure of attempted cannulation due to malignancy have raised concern. Percutaneous transhepatic biliary drainage (PTBD) has become an alternative procedure in most obstructive jaundice cases due to malignancy [3]. This procedure can also be an option for critically ill patients or elderly patients who have comorbidities, even though ERCP is quite safe to be performed in the elderly $[4,5]$. In the 1980s, endoscopic ultrasound (EUS) was introduced as an addition to ERCP procedures to provide a better imaging of the etiology of the disease. Until recently, it has also been able to provide a tissue sample for confirming the diagnosis $[1,6]$. Recently, there have been publications about a rendezvous technique using EUS for biliary drainage when ERCP has failed $[7,8]$. The role of diagnostic and therapeutic EUS in developing countries is still debatable regarding availability, cost, and training curriculum [9]. In Indonesia, the cost for an EUS procedure ranges between USD 1,000 and 2,000, and there is no proper training curriculum yet. So, here, we are reporting several interesting cases among our patients in order to show the impact of EUS in clinical practice.

\section{Method}

The EUS procedure was performed by 2 senior gastroenterologist consultants. One of them is an experienced endosonographer and the other is an EUS trainee who is already experienced in transabdominal ultrasound images and endoscopic procedures. The EUS equipment was an Olympus JF UCT 180 EUS scope which was connected to an Aloka IPF1701C ultrasound machine (Tokyo, Japan). The patients were well sedated before the EUS procedures.

\section{Case Reports}

Case 1

A 52-year-old male was referred from another hospital due to recurrent pancreatitis. The patient had suffered from intermittent abdominal pain for 2 months which radiated to the back. The amylase and lipase levels were 2,890 and 23,802 U/l, respectively, and from the transabdominal ultrasound examination from the other hospital, it was suspected that there might be a stone at the main pancreatic duct. The patient also had already undergone a previous abdominal CT scan at the same hospital, and it was not clear whether there was a stone or calcification at the pancreatic parenchyma without any significant dilatation of the 
duct. Because of unclear imaging findings, EUS was then performed, and it showed a stone inside the parenchyma at the head of the pancreas which was closed to the duct and caused the pancreatic duct compression. EUS also showed that there was lobulation with strands of the pancreatic head parenchyma, suggesting chronic pancreatitis. Then, the patient directly underwent ERCP, and the pancreatic duct could be cannulated even though there was a little bit of resistance when the guide wire was inserted. Based on the pancreatogram, there was no stone and duct dilatation. We decided to perform sphincterectomy and a 5-Fr pancreatic stent was placed. Four hours after ERCP, a repeated measuring of the amylase and lipase levels showed a significant decrease ( 640 and $584 \mathrm{U} / \mathrm{l}$, respectively), and there was no more significant abdominal pain. After that, the patient was discharged, and there was no significant pain anymore during 1 month of follow-up, and the amylase and lipase levels were 34 and $35 \mathrm{U} / \mathrm{l}$, respectively (fig. 1).

Case 2

A 70-year-old female was referred from another hospital with recurrent colicky abdominal pain and a history of jaundice and red-colored urine. She had been diagnosed with nonviral chronic liver disease and idiopathic thrombocytopenic purpura many years ago. A previous transabdominal ultrasound examination only showed multiple cholelithiasis without any significant bile duct dilatation. The patient had also undergone magnetic resonance cholangiopancreatography (MRCP) when she had jaundice, which showed that there was a stone inside the bile duct. The amylase and lipase levels were 50 and $55 \mathrm{U} / \mathrm{l}$, respectively. During follow-up before the patient was referred, the total bilirubin level had gradually decreased from 13.2 to $2.7 \mathrm{~g} / \mathrm{dl}$. The pain had also slightly improved. Then, we performed EUS to evaluate the bile duct and see whether the stone was still there or not, since it is not practical to repeat MRCP for follow-up, especially in the elderly, and in some cases, it is possible to have spontaneous passage of the stone. Our EUS examination revealed that there was still a stone inside the nondilated common bile duct (CBD) with multiple cholelithiasis. So we performed ERCP 1 day after that, and we could successfully extract the large CBD stone. However, when we performed EUS, there were also large esophageal varices, suggesting that the patient also had portal hypertension (fig. 2).

Case 3

A 53-year-old male was referred with obstructive jaundice and dilated CBD due to an unknown cause based on transabdominal ultrasound. The patient had just undergone cardiac stenting about 1 year ago and was still on oral anticoagulants. An abdominal CT scan revealed that there was a pancreatic head mass with a diameter of $7 \times 5 \times 7 \mathrm{~cm}$. Both CBD and pancreatic duct were dilated. It was also reported that there was no lymph node enlargement or liver metastasis. The CA 19-9 level was $392.61 \mathrm{U} / \mathrm{ml}$. The amylase and lipase levels were 45 and $88 \mathrm{U} / \mathrm{l}$, respectively, and the total bilirubin level was $7.97 \mathrm{mg} / \mathrm{dl}$. We performed EUS in this patient, which showed total obstruction at the distal CBD, suggesting that it might be difficult to attempt ERCP cannulation. We also performed fine needle aspiration (FNA). The FNA procedure was successful, and there was no significant bleeding complication even though the patient was still on oral anticoagulants. Based on EUS examination, there was an enlargement of the lymph node, and it seemed that the tumor had infiltrated the portal vein. Then, the patient underwent PTBD, since the guide wire could not pass through the distal CBD for the rendezvous technique. The result of FNA from the mass at the pancreatic head was adenocarcinoma. We decided that this patient would undergo only palliative treatment (fig. 3). 
Lesmana et al.: Impact of Endoscopic Ultrasound Procedures in Various

Pancreatobiliary Disorders in Indonesia Based on a Case Series in a Private Hospital

Case 4

A 15-year-old female came to our unit with recurrent abdominal pain which was located at the epigastrium. The amylase and lipase levels were 129 and $241 \mathrm{U} / \mathrm{l}$, respectively. The highest amylase and lipase levels were 619 and 1,498 U/l, respectively. She had no jaundice, and a transabdominal ultrasound examination revealed that the pancreatic duct was dilated without any clear etiology. Since EUS could provide a better image of the pancreas than a CT scan or MRI, we decided to perform the EUS procedure. The EUS images showed pancreas divisum with chronic pancreatitis. The patient also underwent abdominal CT scan examination to confirm other possible abnormalities, which revealed pancreas divisum (fig. 4).

Case 5

A 59-year-old male came to our unit due to obstructive jaundice with a total bilirubin level of $24.17 \mathrm{mg} / \mathrm{dl}$. There was no clear etiology based on transabdominal ultrasound. It only showed dilated intra- and extrahepatic bile duct without any suspicion of mass either at the pancreatic head region or the distal CBD. The CA 19-9 level was normal. Then, the patient underwent ERCP for biliary stenting. After inserting a plastic stent, the clinical condition became better and the jaundice resolved (the total bilirubin level gradually decreased below the normal limit). EUS was performed several days after the patient had been discharged from the hospital. Our findings showed a mass at the distal CBD, and the FNA result showed only inflammation, and no malignant cells were found (fig. 5).

\section{Discussion}

The need of EUS has been increasing in recent years. When reviewing the past history of EUS, it had almost gone out of use, since the significant impact in daily practice had not been clear. To our knowledge, this is the first report of an EUS case series in a private center which shows the impact of performing the EUS procedure in Indonesia. These several interesting cases could change the paradigm of the need of EUS in our country, since Indonesia is one of the developing countries where cost and investment always become important issues. The initial setup and investment are important, since it is more expensive than the usual endoscopy equipment. The dedicated person who performs this procedure is also important, as learning EUS would need patience, experience, and a good mentor.

The first case shows how an EUS image provides accurate information regarding the proximal pancreatic duct obstruction and the pancreatic parenchyma with a stone inside the small branch and helps to make a more accurate decision for the ERCP procedure. Based on the literature, EUS provides more diagnostic accuracy compared to abdominal CT scanning when used to show the pancreatic parenchyma and its small branch duct [10]. Other imaging modalities such as MRCP could also provide the same accuracy as EUS; however, the length of examination time and the patient's cooperation have become additional issues.

The second case is a typical case of bile duct obstruction due to CBD stone. However, with regard to the age of the patient and the fact that it was unpractical to repeat MRCP just to know whether the stone has passed or not in an insignificant dilatation of the CBD, EUS could provide more accurate information on whether there was still a stone left inside the CBD or not. EUS procedure information can also avoid an unnecessary ERCP procedure, which has several potential complications such as bleeding, pancreatitis, and cholangitis. Attila and Faigel [11] have shown the safety and feasibility of the EUS procedure in the elderly. Our second case also shows the safety of performing EUS in patients with large esophageal varices due to portal hypertension. 
Lesmana et al.: Impact of Endoscopic Ultrasound Procedures in Various

Pancreatobiliary Disorders in Indonesia Based on a Case Series in a Private Hospital

The third case was a patient with obstructive jaundice due to pancreatic head malignancy in whom the EUS image could provide better information than abdominal CT scanning in showing that the mass had already infiltrated the portal vein, and it could also provide information about the total obstruction of the distal CBD, which helped to decide that PTBD might be a better alternative procedure than ERCP. This staging accuracy has also been reported [12]. We know that the length of attempted cannulation time during an ERCP procedure will increase the risk of pancreatitis.

Case 4 was a young female with idiopathic recurrent acute pancreatitis, and the EUS image clearly showed pancreas divisum. The last case was a middle-aged man with obstructive jaundice due to unclear etiology based on transabdominal ultrasound.

In the last 2 cases, the EUS procedure could provide better information about the disease etiology, especially in idiopathic recurrent pancreatitis, and a biopsy could also be performed at the same time if needed, unlike with other imaging modalities such as MRI or CT scanning [13].

\section{Conclusions}

Based on these case series, the EUS procedure has shown a significant impact in clinical practice, especially in establishing the diagnosis of pancreatobiliary disorders when compared to other imaging modalities. It should be considered as an additional tool in gastroenterology practice. The cost, investment, and training will become important issues for EUS development in most developing countries.

\section{References}

1 Mesenas SJ: Does the advent of endoscopic ultrasound (EUS) sound the death knell for endoscopic retrograde cholangiopancreatography (ERCP)? Ann Acad Med Singapore 2006;35:89-95.

-2 Adler DG, Baron TH, Davila RE, Egan J, Hirota WK, Leighton JA, et al: ASGE guideline: the role of ERCP in diseases of the biliary tract and the pancreas. Gastrointest Endosc 2005;62:1-8.

-3 Zhao XQ, Dong JH, Jiang K, Huang XQ, Zhang WZ: Comparison of percutaneous transhepatic biliary drainage and endoscopic biliary drainage in the management of malignant biliary tract obstruction: a meta-analysis. Dig Endosc 2015;27:137-145.

4 Mohammad Alizadeh AH, Afzali ES, Shahnazi A, Sanati A, Mirsattari D, Zali MR: Utility and safety of ERCP in the elderly: a comparative study in Iran. Diagn Ther Endosc 2012;2012:439320.

5 Chang WH, Lei WY: Endoscopic retrograde cholangiopancreatography in elderly patients. Int J Gerontol 2007;1:83-88.

6 Larghia A, Petroneb MC, Galassoa D, Arcidiaconob PG: Endoscopic ultrasound in the evaluation of pancreatobiliary disorders. Dig Liver Dis 2010;42:6-15.

7 Iwashita T, Doi S, Yasuda I: Endoscopic ultrasound-guided biliary drainage: a review. Clin J Gastroenterol 2014;7:94-102.

8 Giovannini M, Bories E: EUS-guided biliary drainage. Gastroenterol Res Pract 2012;2012:348719.

-9 Ahmed F: Endoscopic ultrasonography: challenges and opportunities in the developing world. World J Gastrointest Pharmacol Ther 2014;5:55-56.

10 Nair R, Lawler L, Miller MR: Chronic pancreatitis. Am Fam Physician 2007;76:1679-1688.

11 Attila T, Faigel D0: Endoscopic ultrasound in patients over 80 years old. Dig Dis Sci 2011;56:3065-3071.

$\$ 12$ Jenssen C, Siebert C, Gottschalk U: The role of endoscopic ultrasound in M-staging of gastrointestinal and pancreatobiliary cancer. Video Journal and Encyclopedia of GI Endoscopy. http://dx.doi.org/10.1016/S2212-0971(13)70047-5.

-13 Jenssen C, Von Lampe B, Kahl S: Endoscopic ultrasound in acute pancreatitis. Video Journal and Encyclopedia of GI Endoscopy. http://dx.doi.org/10.1016/S2212-0971(13)70241-3. 
Lesmana et al.: Impact of Endoscopic Ultrasound Procedures in Various

Pancreatobiliary Disorders in Indonesia Based on a Case Series in a Private Hospital
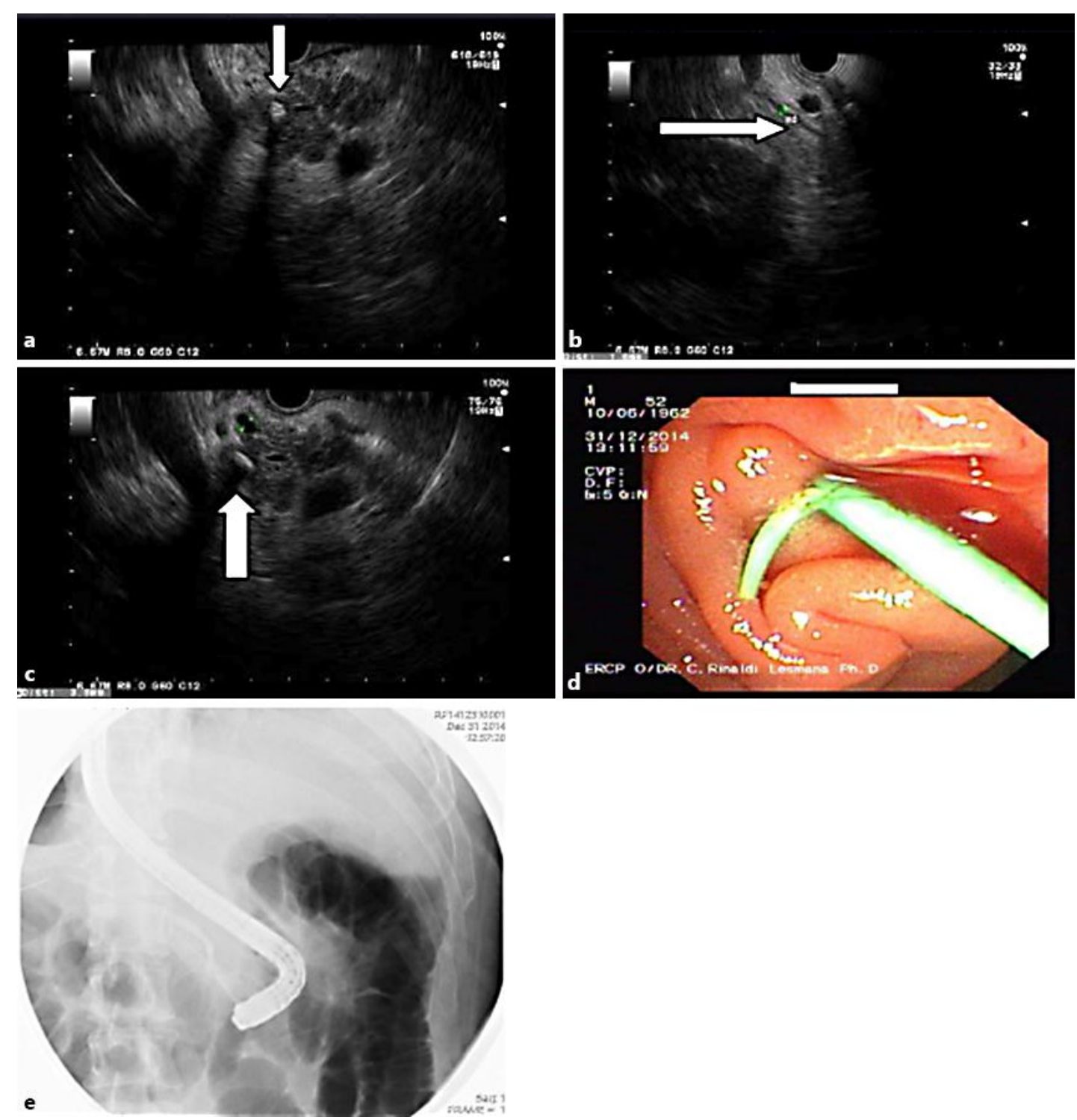

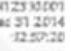

Fig. 1. a EUS image showing suspicion of a pancreatic stone with the differential diagnosis of calcification. b EUS image showing the normal pancreatic duct. c EUS image showing a possible cyst with a stone inside. d Endoscopy image showing that the pancreatic stent was inserted into the pancreatic duct. e Fluoroscopy image during ERCP procedure showing the pancreatogram. 
Lesmana et al.: Impact of Endoscopic Ultrasound Procedures in Various

Pancreatobiliary Disorders in Indonesia Based on a Case Series in a Private Hospital
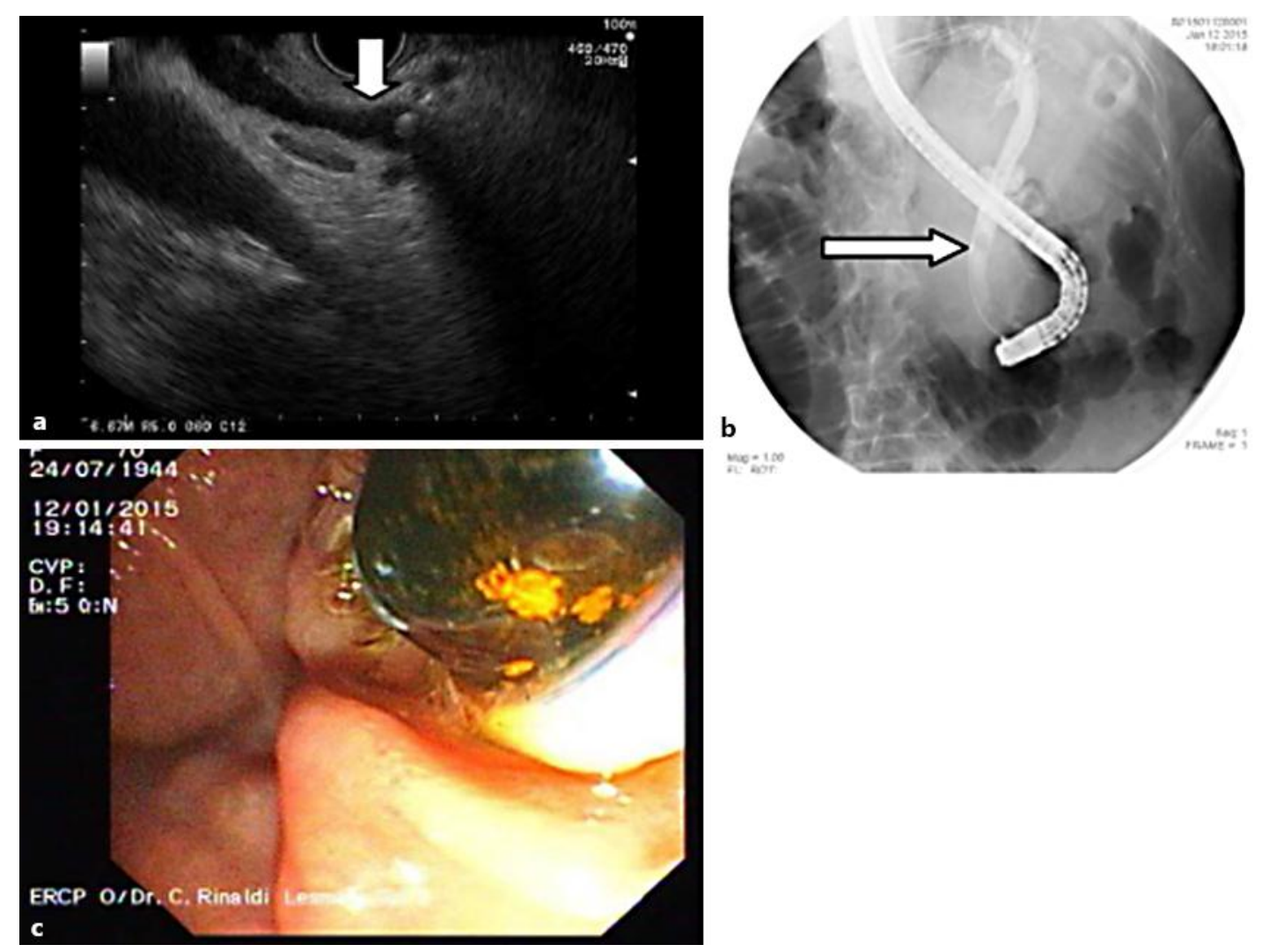

Fig. 2. a EUS image showing a stone inside the normal caliber of the CBD. b Fluoroscopy image during ERCP procedure showing the normal caliber of the CBD with a stone in situ. c Endoscopy image showing that a large stone was extracted through the papilla. 
Lesmana et al.: Impact of Endoscopic Ultrasound Procedures in Various

Pancreatobiliary Disorders in Indonesia Based on a Case Series in a Private Hospital
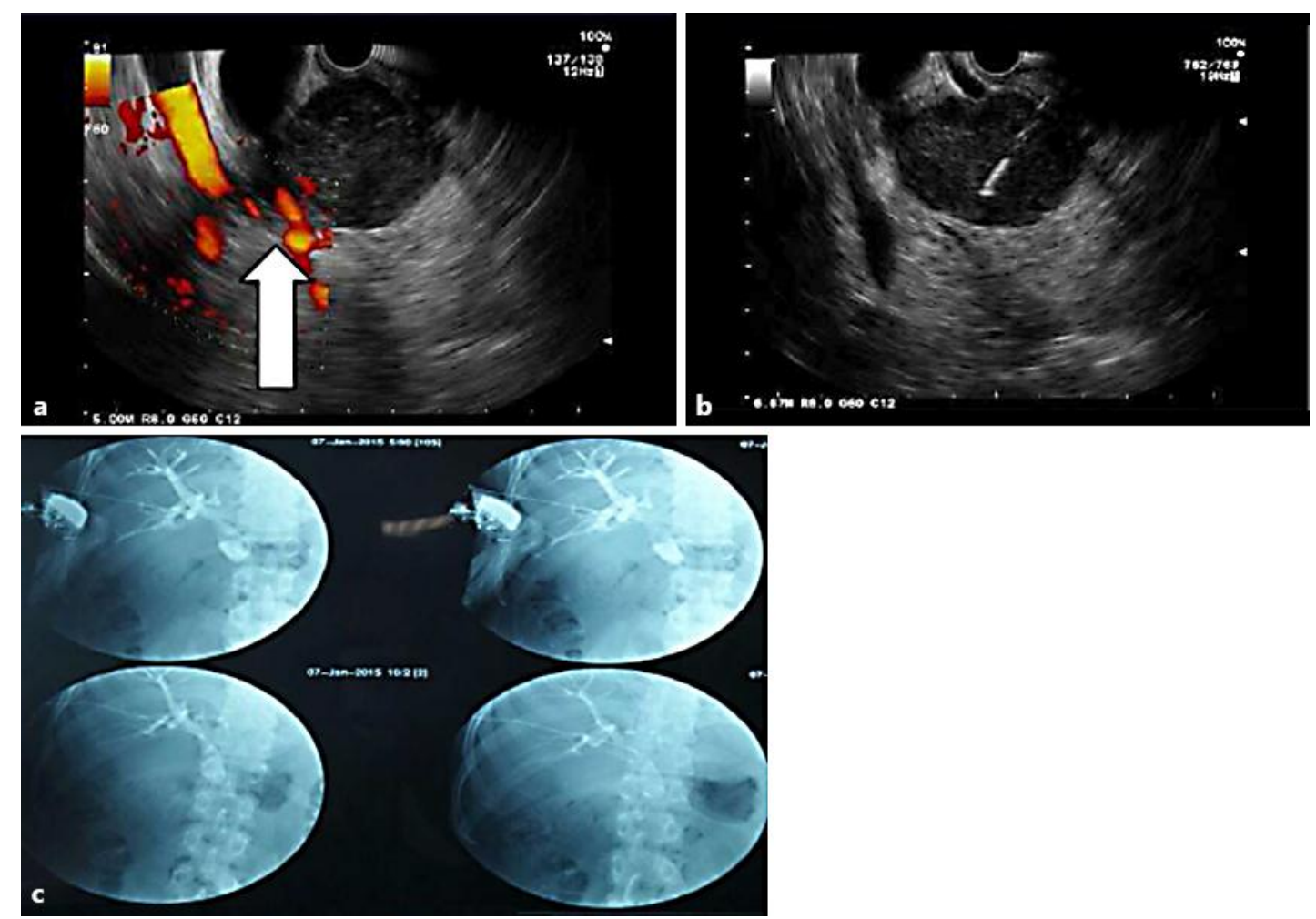

Fig. 3. a EUS image showing the pancreatic head mass that infiltrated the portal vein (Doppler image). b EUS image showing the FNA procedure. c PTBD images using fluoroscopy.
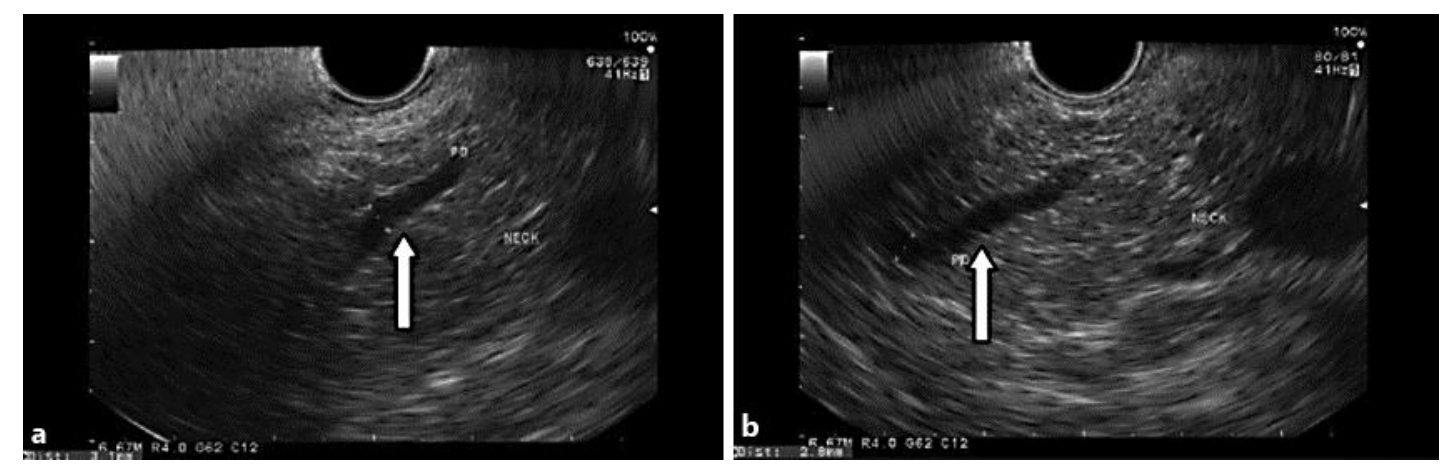

Fig. 4. a, b EUS images showing an abnormal pancreatic duct at the neck through the head of the pancreas, suggesting pancreas divisum. 
Case Reports in
Gastroenterology

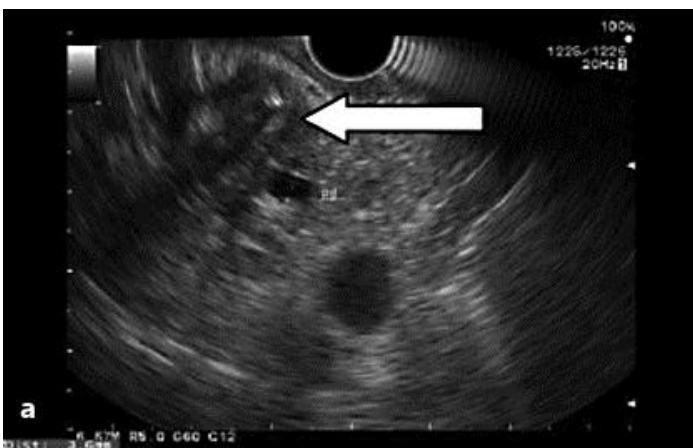

Fig. 5. a, b EUS images showing the distal CBD mass.

Lesmana et al.: Impact of Endoscopic Ultrasound Procedures in Various

Pancreatobiliary Disorders in Indonesia Based on a Case Series in a Private Hospita

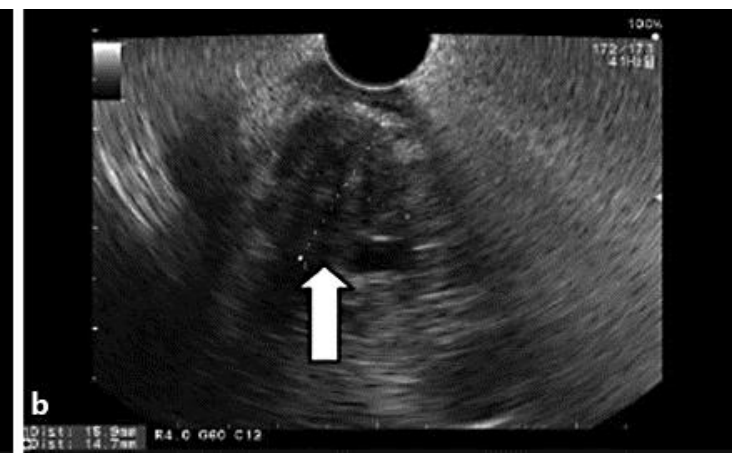

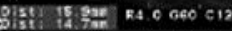

(c) 2015 S. Karger AG, Basel

(c) 2015 S. Karger $A G$,
www.karger.com/crg 